\title{
Two-dimensional burst construction with QoS assurance for OFDMA mobile WiMAX system
}

\begin{abstract}
IEEE 802.16 OFDMA systems have gained much attention for their ability to support high transmission rates and broadband services. For multiuser environments, IEEE 802.16 OFDMA systems require a resource allocation algorithm to use the limited downlink resource efficiently. One of the main problems to increase the resource utilization and throughput is how to construct two-dimensional blocks that realize time and frequency dimensions to multi-users. However, mapping a rectangular shape (i.e. two-dimensional burst) that not enough to utilize some flows in downlink frame, decreases total throughput and is crucial for Quality of Service (QoS) that support real-time applications. In this paper, we propose a low complexity algorithm called Best Edge Block Oriented (BEBO) which complies with 802.16 downlink burst characteristics with assurance of real time throughput. The proposed algorithm reduce the unserved slots by assembling bursts in the edge of the free spaces for reducing outdoor spaces from other bursts; and enlarge the internal burst size gradually to minimize the internal free spaces for a given request size with priority level and channel state information considerations. Simulation results show that the burst construction mechanism result is competitive.
\end{abstract}

